\title{
Herramienta web de ayuda a la gestión de los embalses en España
}

https://doi.org/10.31978/639-19-010-0.623

\author{
Inmaculada Abia Llera' ${ }^{1}$ (iabial@aemet.es) \\ José Voces Aboy² (jvocesa@aemet.es) \\ Eroteida Sánchez García² (esanchezg@aemet.es) \\ Beatriz Navascués Fernández-Victorio ${ }^{3}$ (bnavascuesf@aemet.es) \\ Ernesto Rodríguez Camino ${ }^{4}$ (erodriguezc@aemet.es) \\ María Nieves Garrido del Pozo ${ }^{1}$ (mgarridod@aemet.es) \\ María Concepción García Gómez ${ }^{5}$ (MCGGomez@magrama.es) \\ José Adolfo Álvarez González 6 (jaalvarez@chebro.es) \\ Fernando Pastor Argüello ${ }^{5}$ (FPastor@magrama.es) \\ ${ }^{1}$ AEMET / Delegación Territorial en Castilla y León \\ ${ }^{2}$ AEMET / Delegación Territorial en Cantabria \\ ${ }^{3}$ AEMET / Dirección de Estrategia, Planificación y Desarrollo Comercial \\ ${ }^{4}$ AEMET / Dirección de Producción e Infraestructuras / Área de Evaluación y Modelización del Clima \\ ${ }^{5}$ Dirección General del Agua \\ ${ }^{6}$ Confederación Hidrográfica del Ebro
}

\begin{abstract}
RESUMEN
La herramienta web diseñada para apoyar la gestión de los embalses en España, es fruto del equipo multidisciplinar formado en S-ClimWaRe, el servicio climático cuyo objetivo es avanzar en la gestión del agua en España.

En el presente artículo se explicarán sus dos facetas, diagnóstico y pronóstico. La primera permite al usuario explorar, sobre un grid o en cualquier embalse, el riesgo y la variabilidad hidrológica ligados a la variabilidad climática. Esto se lleva a cabo por medio de un conjunto de diagnósticos obtenidos de las series temporales de observaciones hidrológicas y meteorológicas y un conjunto de «drivers» o impulsores climáticos. La otra faceta de esta herramienta se refiere al ámbito de la predicción, suministrando pronósticos estacionales que incluyen la pericia del sistema.
\end{abstract}

PALABRAS CLAVE: predicción; pronóstico; estacional; servicio climático; NAO; gestión; agua.

\section{INTRODUCCIÓN}

Dentro del Marco Mundial para los Servicios Climáticos impulsado por la Organización Meteorológica Mundial, se ha desarrollado el servicio climático S-ClimWaRe (por sus siglas en inglés, Seasonal Climate predictions to support Water Reservoirs management in Spain) con el objetivo de mejorar la gestión de los embalses en España a través del uso de la información meteorológica climática y estacional.

Este servicio surgió inicialmente como un caso de estudio dentro del proyecto EUPORIAS para difundir en la sociedad el uso de las predicciones estacionales y decadales. Tras el final del proyecto y debido a la utilidad que se está obteniendo del mismo, el servicio climático ha continuado desarrollando sus actividades. 
El grupo responsable del desarrollo de S-ClimWaRe está formado por:

- La Agencia Estatal de Meteorología (AEMET), el servicio meteorológico del gobierno de España.

- La Dirección General del Agua, órgano del Ministerio para la Transición Ecológica encargado de la gestión del agua.

- Las Confederaciones Hidrográficas del Duero, Ebro, Miño-Sil y Tajo, que son los organismos encargados de la gestión directa del agua en estas cuencas españolas.

Cada una de estas instituciones pone su contribución para formar un equipo multidisciplinar de meteorólogos, científicos, ingenieros y gestores del agua que avanza en el conocimiento de los procesos que determinan el comportamiento de los recursos hídricos.

El trabajo del grupo se coordina y progresa a través de reuniones periódicas, talleres de trabajo presenciales, seminarios de formación en línea, etc. Así se consigue un intercambio de ideas muy productivo y enriquecedor para todos los miembros. Entre los resultados obtenidos están el diseño, la implementación y la evaluación de diferentes sistemas de predicción estacional del volumen de agua que entra en los embalses, sistemas de gestión del agua y herramientas para el análisis y comprensión de la variabilidad hidrológica y climática.

Y es de estas últimas herramientas de lo que se va a hablar en este artículo; concretamente de la web diseñada en S-ClimWare para reunir y poner a disposición de los usuarios el conjunto de instrumentos que permiten analizar el comportamiento de los recursos hídricos en España que está ligado a la variabilidad del clima.

\section{HERRAMIENTA WEB DE S-ClimWaRe}

Como se ha indicado en la introducción, en el servicio climático se han desarrollado una serie de herramientas para ayudar a analizar y comprender la variabilidad que afecta a los recursos hídricos. Para evitar que la complejidad de estas herramientas o la dificultad de acceso a las mismas limiten el uso por parte del usuario se ha diseñado esta web en forma de caja de herramientas.

En conjunto, la web proporciona a los expertos y no tan expertos una manera eficaz de explorar las relaciones entre variables como precipitación o aportaciones a los embalses y algunos drivers climáticos como la Oscilación del Atlántico Norte (NAO). De esta forma, el portal se constituye en un instrumento fundamental en el apoyo a los procesos de decisión en la gestión del agua.

En el proceso de diseño del sistema ha estado implicado todo el grupo de trabajo constituido en S-ClimWaRe manteniéndose siempre centrado en las necesidades de los usuarios potenciales. La implementación y el mantenimiento corren a cargo de AEMET.

Esta caja de herramientas se ha diseñado teniendo en mente su utilidad y accesibilidad de forma que es escalable y modular. De esta manera, es posible incorporar con facilidad nuevas herramientas, índices y variables, y retirar los aspectos que se vayan quedando desfasados. Además, al estar basado en una web el usuario tiene la seguridad de estar accediendo a la última versión con el contenido actualizado.

Antes de empezar a hablar de los datos y las relaciones entre las variables y los drivers, hay que señalar que, a día de hoy, la predecibilidad a escala estacional en la península ibérica es bastante limitada, verificándose que hay una relación entre la NAO invernal (diciembre, enero y febrero) y la precipitación/aportaciones del invierno extendido (de noviembre a marzo). Más información sobre este punto puede encontrarse en BRANDS et al., 2012 y en COHEN y Jones, 2011. Es por esto que en la web se exploran las relaciones entre estos parámetros en estos periodos ya que son los que proporcionan información de utilidad en la gestión de los embalses. 
Por otra parte los datos que alimentan al sistema son los siguientes:

- Datos de aportaciones de agua a los embalses, que provienen de la Dirección General del Agua. Abarcan a todos los embalses situados en el territorio peninsular de España, aunque la longitud de la serie de datos depende de cada embalse.

- Datos de precipitación provistos por AEMET. Son datos basados en una rejilla de $5 \mathrm{~km}$ de resolución sobre la Península y Baleares y el periodo va desde 1951 a 2017 (PERAl et al., 2017).

- Datos del estado de la NAO, que provienen del índice estandarizado que publica la National Oceanic and Atmospheric Administration (NOAA).

El sitio web se organiza en dos vistas, la vista de diagnóstico y la de predicción. La selección de cada una se hace en los selectores de la esquina superior izquierda (véase figura 1). Se describe a continuación el contenido de la web haciendo hincapié en las herramientas ya que el funcionamiento está descrito en la ayuda del propio sitio.

\subsection{Vista de diagnóstico}

En la figura 1 podemos ver la vista de diagnóstico.

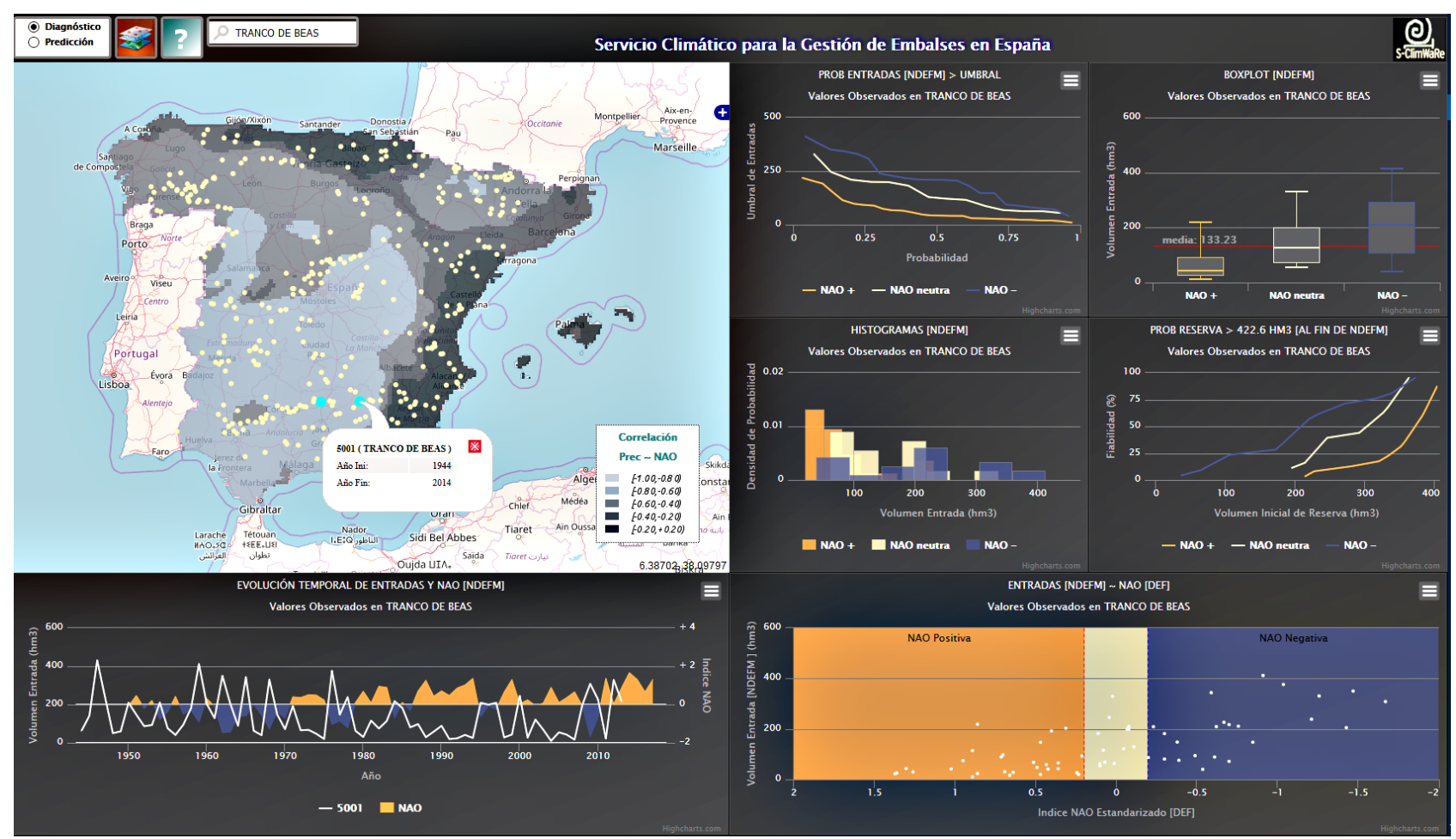

Figura 1. Vista de diagnóstico.

En esta vista se incluyen las herramientas que permiten al usuario explorar en cualquier embalse o punto de grid la variabilidad hidrológica y el riesgo asociado a la variabilidad climática. Se trata de un conjunto de diagnósticos obtenidos a partir de las series temporales de las observaciones de la precipitación, las aportaciones y la NAO, centrándose cada elemento mostrado en una característica de las relaciones existentes.

Esta vista está constituida por

- Un panel principal donde se encuentra un mapa para seleccionar el punto a examinar. Sobre este mapa se pueden hacer las operaciones habituales (seleccionar el tipo de mapa, zoom, activar y desactivar capas) para adaptar la visibilidad a nuestras necesidades. Hay también un selector de cuencas y subcuencas 
para mostrar los límites de las mismas y facilitar la identificación a los usuarios. En este panel aparecen los embalses como puntos amarillos y, de fondo, aparece la primera herramienta, la correlación entre la NAO y la precipitación invernal. Seleccionando un embalse con el ratón, el resto de herramientas que aparecen en la ventana se actualizan con los datos del embalse seleccionado en el mapa. Si se pincha sobre cualquier punto que no sea un embalse, se actualizan con los datos de precipitación del punto de grid más cercano. Esto nos permitirá investigar cómo es la relación NAO-aportación en el caso de un embalse o cómo es la relación NAO-precipitación en el caso de cualquier otro punto.

- Evolución temporal de la variable con la NAO. Debajo del panel principal de selección, se sitúa el gráfico que muestra la evolución temporal de la variable en cuestión (precipitación o aportación al embalse) junto con la evolución de la NAO. De esta forma es posible ver la relación temporal que existe entre el driver climático y la variable. La NAO se separa en tres intervalos (positiva, neutra y negativa) para facilitar su visualización. Este criterio se sigue en el resto de gráficos de la web. Y al igual que en el resto de herramientas, estas son activas, de forma que al pasar el puntero sobre ellas se visualizan los datos, se pueden activar/desactivar diversos elementos y los gráficos pueden imprimirse o descargarse en varios formatos.

- Diagrama de dispersión. Se sitúa en la parte inferior derecha y muestra el índice NAO (eje de abscisas) frente a la precipitación/aportación (eje de ordenadas). Permite analizar la relación entre la variable y el driver climático.

- Curvas de probabilidad. En la parte superior, inmediatamente a la derecha del panel principal se representa la probabilidad de que la variable supere diferentes umbrales dependiendo del estado de la NAO. Se muestran por tanto tres curvas, una por cada posible estado de la NAO (positiva, neutra o negativa). Si estamos considerando un embalse, en el eje de ordenadas se representan los valores de las aportaciones al mismo y, considerando cada uno de dichos valores como un valor umbral, se calcula la probabilidad de superación de dicho umbral, es decir, el porcentaje de datos sobre el conjunto de datos observados para ese embalse en que las aportaciones son superiores a dicho umbral. Este valor de probabilidad se representa en el eje de abscisas, obteniendo la curva de probabilidad que corresponde uniendo por orden creciente de probabilidad el conjunto de puntos representados.

- Boxplot. Se sitúa a la derecha de las curvas de probabilidad y es una representación de la dispersión de las observaciones en función del estado de la NAO. La caja está definida por los cuartiles primero y tercero, englobando así el $50 \%$ de los datos, y los «bigotes» llegan en este caso hasta el mínimo y el máximo de la distribución. Si el número de datos no es suficiente como para que sea representativo se omite esta herramienta.

- Histograma. Debajo de las curvas de probabilidad y encima del diagrama de dispersión. En realidad son tres histogramas, uno por cada estado de la NAO. Un cierto nivel de transparencia de las barras y el hecho de poder activar y desactivar cada uno mejora la visibilidad de los mismos. Este gráfico permite determinar si, en el punto seleccionado, el driver (en este caso la NAO) es un buen discriminador del comportamiento de la variable escogida.

- Curvas de fiabilidad. Esta herramienta se muestra debajo del boxplot y encima del diagrama de dispersión. Solo aparece cuando se selecciona un embalse. En el eje de ordenadas se representa la probabilidad de que la reserva al final del invierno extendido (31 de marzo) esté por encima del percentil 75 de la aportación y en el eje de abscisas aparece el volumen inicial de la reserva (al inicio del invierno extendido, el 1 de noviembre). Representa, por tanto, la probabilidad de que dada una reserva inicial en el embalse, al final del periodo el embalse esté «bastante lleno». Dado que esto depende del carácter de la NAO, se representan tres curvas.

\subsection{Vista de predicción}

En esta vista se accede a las predicciones estacionales para la precipitación sobre los puntos del grid de 5 km y para las aportaciones a los embalses. 


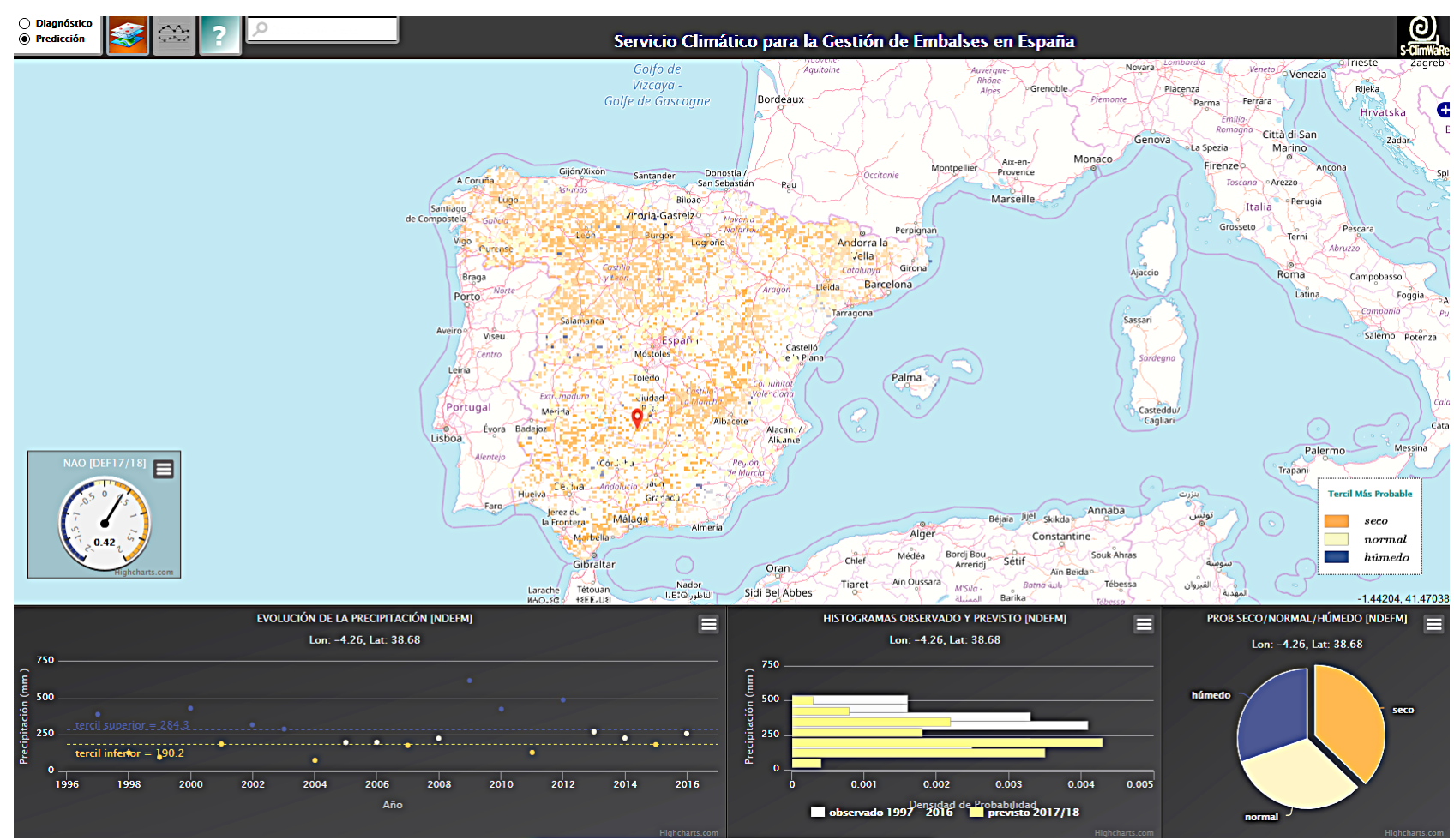

Figura 2. Vista de predicción.

Actualmente se está usando un sistema de predicción estacional estadístico desarrollado por AEMET para S-ClimWaRe. Se trata de un sistema de predicción por conjuntos que en cada punto de grid y embalse genera un conjunto de predicciones (ensemble) de precipitación o de aportaciones. Este sistema ha sido evaluado haciendo una serie de predicciones retrospectivas de forma que se conoce la pericia del mismo en cada punto. Para simplificar la medida de la habilidad del sistema, en la web esta característica viene representada por el área ROC cuyos valores se mueven entre 0 y 1 , de forma que valores próximos a 1 indican un buen sistema de predicción y valores inferiores a 0,5 indican que el sistema es peor que el sistema usado para comparar. Los detalles del sistema así como una completa evaluación del mismo se pueden encontrar en Voces et al., 2016.

\section{En esta vista encontramos}

- Un panel principal similar al de la vista de diagnóstico y con el mismo propósito, seleccionar el punto/embalse sobre el que queremos ver los pronósticos. A diferencia del anterior, en este caso el fondo está ocupado por una rejilla de píxeles de $5 \mathrm{~km}$ coloreados según el tercil de precipitación más probable. Una forma eficaz de transmitir las predicciones estacionales consiste en dar la probabilidad de cada uno de los 3 terciles de la serie de observación. En este mapa se representa en naranja el primer tercil (por debajo de lo normal o seco), en ocre el normal y en azul el tercil superior. Además, cada pixel tiene un nivel de transparencia en función de la pericia que el sistema de predicción demuestra tener sobre él. Cuanto más fiable sea el pronóstico en un punto, más denso (menos transparente) será el color del mismo. De esta forma, de un vistazo, en cada punto, se ve el pronóstico más probable junto con la confianza que podemos tener en él. Los pronósticos con poca agudeza (sharpness) (la probabilidad de los 3 terciles es muy similar) tampoco se muestran dada la poca información que aportan frente a la climatología. Por último, notar que en la esquina inferior izquierda de este panel aparece un gráfico de tipo reloj que indica el pronóstico determinista de la NAO invernal.

- Serie temporal. Debajo del panel principal, en el lado izquierdo aparece la serie temporal de la precipitación/aportación sobre el punto/embalse seleccionado. Cada punto de la gráfica está rotulado según el tercil al que pertenece y también aparecen dos líneas punteadas que indican los valores del tercil inferior y 
superior. De esta forma tenemos una referencia para poder comparar el pronóstico y saber qué significan en este punto los términos «seco», «normal» y «húmedo».

- Histograma observado y previsto. Se sitúa inmediatamente a la derecha de la serie temporal. Aquí aparecen representados el histograma con la distribución de las observaciones históricas (en blanco) y el histograma con la distribución de la predicción por conjuntos (en amarillo). Tenemos así una visión rápida de dónde está el pronóstico con respecto a la serie histórica.

- Gráfico de probabilidad. A la derecha del anterior vemos en forma de diagrama de tarta las probabilidades de los 3 terciles. Pasando el puntero por encima aparece el valor de la probabilidad y el valor de la pericia del sistema.

\section{CONCLUSIONES Y PRÓXIMOS PASOS}

Con esta utilidad se ha conseguido poner a disposición de los usuarios, de una forma sencilla y eficaz, la información necesaria para ayudar en el proceso de toma de decisión en las tareas relacionadas con la gestión de los recursos hídricos en España.

La web se ha construido en el marco de un servicio climático en el que se sigue trabajando y avanzando. Teniendo en cuenta esto, se ha diseñado un producto flexible en el que es relativamente sencillo incorporar nuevas variables, drivers, herramientas o sistemas de predicción de forma que los avances realizados en S-ClimWaRe se pueden incorporar con facilidad para que lleguen al usuario.

Actualmente, en el marco de la participación de S-ClimWaRe en el proyecto MEDSCOPE, se está trabajando en esta línea, buscando mejorar la pericia de la predicción estacional y extendiéndola a otros periodos y a otras variables.

\section{REFERENCIAS}

Brands, S., Manzanas, R., Gutiérrez, J. M. y Cohen, J., 2012. Seasonal Predictability of Wintertime Precipitation in Europe Using the Snow Advance Index. J. Climate, 25, 4023-4028. doi: 10.1175/JCLI-D12-00083.1.

Cohen, J., y Jones, J., 2011. A new index for more accurate winter predictions. Geophys. Res. Lett., 38, L21701, doi: 10.1029/2011GL049626.

Peral, C., Navascués, B., y Ramos, P., 2017. Serie de precipitación diaria en rejilla con fines climáticos. Nota Técnica $n^{\circ} 24$ AEMET. http://www.aemet.es/documentos/es/conocermas/recursos_en_linea/ publicaciones_y_estudios/publicaciones/NT_24_AEMET/NT_24_AEMET.pdf.

Voces, J., Sánchez García, E., Navascués, B., Franco, F. y Rodríguez Camino, E., 2016. Sistema estadístico de predicción estacional para la gestión de los embalses en España. Nota Técnica n ${ }^{\circ} 21$ AEMET. http:// www.aemet.es/es/conocermas/recursos_en_linea/publicaciones_y_estudios/publicaciones/detalles/ NT_21_AEMET.pdf. 